
\title{
R Research Soure \\ Scientifically Speaking We know a lot, or do we? A model of knowledge using the Electromagnetic Spectrum
}

Fernando Espinoza ( $\sim$ phyfze@hofstra.edu )

Hofstra University https://orcid.org/0000-0002-8503-3885

\section{Research Article}

Keywords: Science Education, Modeling, Scale of measurements, Misconceptions, Use of the Spectrum, Epistemology

Posted Date: June 28th, 2021

DOl: https://doi.org/10.21203/rs.3.rs-660708/v1

License: (c) (1) This work is licensed under a Creative Commons Attribution 4.0 International License.

Read Full License 


\title{
Scientifically Speaking We know a lot, or do we? A model of knowledge using the Electromagnetic Spectrum
}

\begin{abstract}
A quantitative model of human knowledge of the physical world based on the use of various parts of the electromagnetic spectrum is proposed. The model is epistemologically effective in demonstrating limits to what scientific knowledge can provide us about the physical world, thus enhancing for students the potentially exploratory opportunities available in scientific research, based on how little we really know. Additionally, the model provides a pedagogically useful way to engage students in considerations of the role of measurement, scale and estimation; these features are urgently needed by many students according to international assessments and are particularly important for non-science majors.
\end{abstract}

Keywords: Science Education, Modeling, Scale of measurements, Misconceptions, Use of the Spectrum, Epistemology.

\section{Introduction}

Science education in general, and particularly physics instruction appears to many nonscience students as predominantly a body of knowledge, which perhaps contributes to its perceived difficulty and other intimidating features. To encourage interest and participation in scientific careers among students, and to ensure that the public-at-large sees the value of scientific knowledge, we need to develop an appreciation of what science can and cannot do for us, according to national science education organizations.

Part of that appreciation involves a recognition of science's limits in terms of knowledge of the natural world. The objective of this essay is to use parts of the electromagnetic spectrum to develop a model of human knowledge of the physical world. Such a model helps convey how little we really know, something that many students find surprising about scientific knowledge.

There are many accounts including those based on statistics that show most children around the world find mathematics and science hard, and so these tend not to become their favorite subjects; however, one of the fundamental aspects of an education is to learn those important fields, as we consider them essential for critical thinking and a well-informed citizenry.

There is another perception originating early in education that science is just not for everybody. One of the reasons many students show little interest in science is that adults themselves are not excited about it. In addition, most students that begin to study science will stop studying it in earnest before they even get to college.

For many students, the perception of most teaching of science is about information considered factual; in other words, what we know about the natural world. Previous Pew Research Center knowledge surveys have shown that young people are poorly informed about current events 
and politics; although, curiously, this does not seem to be the case with science knowledge. Interestingly, those younger than 30 years old score higher on knowledge tests than do those 65 years and older. Nevertheless, the best-informed people about science, according to the results of a science quiz, are those 30 to 49 years old. Additionally, the majority of people in the US believe that science has had a positive effect on society (Pew Research Center).

One aspect of the need to improve the state of science education is to place more emphasis on how scientific knowledge has come about. This entails a shift from learning about facts, to learning about processes. The history of science illustrates that an understanding of processes is more important than knowledge about facts, particularly in the modern age when the rate of progress renders facts obsolete almost as soon as they are available.

The need to address the rapid change in knowledge about the natural world in the modern age has provided ways to quantify the process. One of the earliest attempts contained the realization that scientific data was doubling every 15 years, thus leading to an exponential model of the increase in scientific knowledge (deSolla Price. D, 1965). What began then as the field of Scientometrics, is a way to see relationships between the sociology of scientific knowledge and information sciences (Leydesdorff and Milojevic, 2015). This field then represents an organization of knowledge based on individual productivity and availability of information about scientific fields.

Interestingly, an exponential decrease has also been proposed to model the rate at which known facts become obsolete (Arbesman, S., 2012). The suggestion by Arbesman that eventually such exponential growth in knowledge cannot be indefinite, acknowledges that there are limitations, and this can be represented graphically.

Figure 1 shows two types of growth; part a) indicates an exponential growth towards infinity, part b) shows growth that partially looks exponential but reaches an upper limit.

(Insert Figure 1 here)

Figure 1 Two graphs indicating different types of growth; part a) shows that as time goes on the number grows exponentially, eventually as time continues indefinitely the growth goes towards infinity. Part b) on the other hand shows that despite an initial exponential growth, the overall pattern indicates that an upper limit to the growth is eventually reached

It must be emphasized that the curves shown above can be used to represent human knowledge growth in terms of our ability to discover properties that we attribute nature to possess. However, the left one a) seems unrealistic given a number of features that have been identified to afflict human knowledge (e.g. Godel's incompleteness theorems for mathematical knowledge, limitations of inductive knowledge from experimental results as predicted by quantum mechanics, and human confirmation bias from cognitive psychology, etc.). The specific features mentioned play a role both in our theoretical ability to conceive of models that can be formulated quantitatively, as well as our empirical ability to confirm such models. Consequently, should an acknowledgment of an upper limit to our potential knowledge about nature be a hindrance to scientific progress? Absolutely not, as exemplified by repeated historical instances of predictions about reaching a limit to our knowledge of the physical world. A particular case in point was the 
view in physics towards the end of the nineteenth century before quantum mechanics and relativity revolutionized the field.

Nevertheless, a distinctive ability of scientific knowledge is the fact that one can say something about nature without knowing everything. Therefore, the expectation is that if nature is inexhaustible in its features, we may go a long way towards understanding them. After all, it is unreasonable to conclude that science is anything but a provisional interpretation of natural events; thereby deriving its particular strength from being inconclusive and subject to revision (Espinoza, 2011).

However, a consideration of our knowledge about facts can be useful; particularly in terms of conveying to students how little we really know about the natural world, despite the enormous accumulation of factual knowledge over the ages. This approach was used in The Encyclopedia of Ignorance, a wondrously instructive and engaging book, where all the contributions were made based on the premise that expertise in a given field entails an acknowledgment of what is unknown about it at the time (Duncan \& Weston-Smith, 1977).

Therefore, as a motivator for further consideration and reflection, we ought to avoid underestimating the value of the unknown.

\section{Development of a Model to Illustrate Knowledge of the Natural World}

"The growth in human knowledge during the past 300 years has been largely the result of the growth in scientific knowledge" Sir Karl Popper.

Indeed, much progress has been made in many other areas of knowledge, but nothing comparable to what has been generated by scientific knowledge. However, such an enormous amount of knowledge has been accomplished through two basic types of research-nomological (based on the regularity exhibited by nature that allows for repeatability), and historical (where one studies events and processes that may have occurred only once, or a limited number of times, and so there is a considerable amount of reconstructive speculation).

It is precisely the incredible incremental growth in scientific knowledge that has convincingly made evident the shortcomings of the traditional mode of instruction in science, where the emphasis has been on the transmission of information. The latest reform in science education Next Generation Science Standards (NGSS) identifies the following required practices of science and engineering as essential for all students to learn:

1. Asking questions (for science) and defining problems (for engineering)

2. Developing and using models

3. Planning and carrying out investigations

4. Analyzing and interpreting data

5. Using mathematics and computational thinking

6. Constructing explanations (for science) and designing solutions (for engineering) 


\section{Engaging in argument from evidence}

8. Obtaining, evaluating, and communicating information

Such an emphasis on practices has been accomplished by restructuring the science curriculum so that they can be applied to a set of disciplinary core ideas, thus making the content that students need to master more manageable in its extension.

In fact, a committee of researchers and public and private leaders have declared an urgency in establishing such standards due to the fact that "science and mathematics achievement continues to lag compared to our international competitors, and this lag has already begun to impact the competitiveness of young Americans as well as the competitiveness of the U.S. in the global economy.” (NGSS, 2012).

Therefore, in order for students to develop the needed proficiency with these practices, an element of engagement must accompany our efforts. As stated before many students seem surprised when exposed to the limits of scientific understanding, undoubtedly as a result of their perception that science is predominantly a body of knowledge.

The use of a model can help to demonstrate the amount of knowledge acquired and what is left to explore about the natural world. It consists of using specific parts of the electromagnetic spectrum, and expressing relationships between known constants. However, such constants are not directly evident from most illustrations of the spectrum. As the figure below illustrates, the typical spectrum displayed is limited to a range of wavelengths and frequencies that is too narrow for a determination of the needed relationships between known constants in the development of a model.

(Insert Figure 2 here)

Figure 2 The typical electromagnetic spectrum shows a range of wavelengths that is too narrow to be useful in building the proposed model

Figure 2 illustrates a difficulty in developing a model that takes into consideration the enormous range of wavelengths needed to establish relationships aimed at establishing limits to human knowledge of the natural world. The range of wavelengths shown is only between waves (and the objects that one can investigate with them) of about $1 \mathrm{~km}\left(1 \times 10^{3} \mathrm{~m}\right)$ in length at one end, and $1 \mathrm{pm}\left(1 \times 10^{-12} \mathrm{~m}\right)$ at the other. These correspond to long radio waves at the longest end, and short gamma rays at the shortest. Thus, only a range of $1 \times 10^{15} \mathrm{~m}$ is available.

A much better representation of the range needed is available by using a representation such as the Scale of the Universe (htwins.net). This model is dynamic, and its range can be stretched all the way to describe the shortest and longest imaginable distances, and the objects found at those scales.

To construct the model, we consider the length of the electromagnetic spectrum from the shortest known wavelengths (gamma rays), to the longest known (radio waves). Longer and shorter waves than those depicted in any spectrum can be represented in terms of the symbol for wavelength $\lambda$ approaching either infinity $(\infty)$, or zero $(0)$. Then we select points on the spectrum 
continuum that correspond to known constants. Figure 3 shows how this is accomplished (Espinoza, 2011).

The model can be represented quantitatively as a percentage of knowledge, by the following expression

$$
\mathrm{F}(\%)=\left(\frac{\sigma_{\mathrm{B}-\mathrm{C}}}{\mu_{\mathrm{A}-\mathrm{D}}}\right) \times 100 \%=\frac{100 \%}{\chi}
$$

with $\chi=\frac{\mu_{\mathrm{A}-\mathrm{D}}}{\sigma_{\mathrm{B}-\mathrm{C}}}$ where $\mu$ and $\sigma$ are the estimated and precise parts of the spectrum, with their respective limits (the known constants).

(Insert Figure 3 here)

Figure 3 The electromagnetic spectrum (the dashed line) and the sections selected where our knowledge is highly reliable (B-C), and those where it is not $(<\mathrm{B} \&>\mathrm{C}$ ). Not to scale. (Rowman \& Littlefield publishers, Used by Permission)

Figure 3 shows two regions of the electromagnetic spectrum chosen to indicate the approximate boundaries to those areas where our knowledge of the natural world is well established (B-C), and those where what we know is estimated (A-D). To separate the regions, points $\mathrm{A}, \mathrm{B}, \mathrm{C}$, and $\mathrm{D}$ represent several known constants, and we choose the following generally accepted values:

$\mathbf{A}=$ The Planck Length $\left(\approx 1.6 \times 10^{-35} \mathrm{~m}\right)$, the shortest possible reachable distance

$\mathbf{D}=$ The currently expected size of the observable universe $\left(\approx 3.9 \times 10^{26} \mathrm{~m}\right)$

$\mathbf{B}=$ The Compton Length $\left(\approx 1 \times 10^{-12} \mathrm{~m}\right)$, the limit of resolution of all instruments

$\mathbf{C}=$ The Hubble Length $\left(\approx 1.3 \times 10^{26} \mathrm{~m}\right)$, the limit of astronomically observable distances.

It should be apparent that both points $\mathrm{B}$ and $\mathrm{C}$ determine the limits of instruments that provide us with the tools to explore the very microscopic world at one end, and the astronomically large at the other. It is conceivable that this range will be extended in the future; however, at the present time the range is a valid approximation; as it should also be apparent from the figure, the other points A and D are placed significantly away from both ends of the spectrum.

The range of distance between $\mathrm{B}$ and $\mathrm{C}$ is $1.3 \times 10^{38} \mathrm{~m}$; this corresponds to the part of the universe where what is known is regarded as being highly accurate and reliable. The range of distance between $\mathrm{A}$ and $\mathrm{D}$ is $2.4 \times 10^{61} \mathrm{~m}$, which corresponds to the part of the universe where what we know is estimated. Comparing these two ranges one can see that B-C.

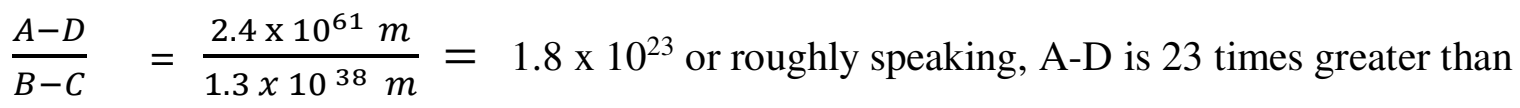

A difference of 23 orders of magnitude between what we know with a high level of accuracy and reliability, and what we estimate about the observable universe, seems nearly 
incomprehensible on first inspection. However, if we use a pie chart to represent the relationship between the amount of knowledge that is highly reliable to that which is estimated, we can assign the range of A-D to be the pie (100\%), and the range B-C to be a slice of it. Therefore, if the pie (A-D) is 23 times greater than the section (B-C), we can divide the pie into 23 parts, resulting in

$$
\frac{360^{0}}{23}=15.6^{\mathrm{o}}
$$

This means that B-C corresponds to a slice that is roughly $4 \%$ of the whole pie $\left(\frac{15.6^{0}}{360^{0}}\right)$. In other words, B-C would represent a slice of A-D approximately equal to $15.7^{\circ}$, or roughly $4 \%$ of the whole pie. We can represent the relationship graphically as in Figure 4 below.

(Insert Figure 4 here)

Figure 4 The two ranges translated as percentages; A-D as the whole pie (100\%), and B-C as the slice (4\%). (Rowman \& Littlefield publishers, Used by Permission)

Figure 4 shows that if the range of what we know with a high level of reliability (B-C) constitutes only $4 \%$ of what is potentially knowable (A-D), then $96 \%$ remains as estimated, and therefore lacking the same level of confidence. It should be noted that points A and D remains strictly in the theoretically possible region, where empirical confirmation at the present time seems out of the question. At these extreme points, our most sophisticated physical theories may exhibit properties that can only be interpreted in simulations.

The model is decidedly optimistic since, as one can see in Figure 2, there are immense regions left on both sides of the spectrum beyond A and D. Therefore, the regions that lie beyond $\mathrm{D}$ and A constitute those parts of the universe that are so currently inaccessible that we don't even know what sorts of questions to ask about phenomena that lie in such regions. Consequently, we must conclude that despite the tremendous scientific advances that have been made, what is actually known about the physical universe with a high level of reliability is really a tiny amount compared to what is potentially available to be investigated.

The significance of this realization lies in its potentially motivating value to encourage interested students to become involved in scientific disciplines, since they could potentially help a great deal in uncovering the secrets that lie in those regions of investigation. In addition, it can provide other students and a wider public with a more realistic picture of what science and scientific knowledge are capable of.

Another useful feature of the electromagnetic spectrum is that we can use it to illustrate the incredible advances made in exploring nature since two very important inventions in the seventeenth century. Both the telescope and the microscope allowed humans to investigate previously unobserved regions of nature. In terms of magnification, we can compare what one could investigate with the naked eye, to what microscopy has made possible. We can use the Scale of the Universe (htwins.net) to obtain the following numbers:

(1) Smallest object visible to the naked eye $1 \times 10^{-4} \mathrm{~m}$

(2) Smallest thing visible to an optical microscope $-2 \times 10^{-7} \mathrm{~m}$ 
(3) Smallest thing visible to an electron microscope- $5 \times 10^{-11} \mathrm{~m}$

(4) Lengths shorter than $1 \times 10^{-16} \mathrm{~m}$ cannot be confirmed

To get a sense of the advances made in magnification since the invention of the microscope, we can divide (1) by (3). We can bypass the limit of resolution of optical microscopes since electron microscopes have extended the range of exploration and investigation by switching from using the wavelength of visible light, to using that of electrons.

$$
\frac{\text { Limit of vision }}{\text { limit of resolution of electron microscope }}=\frac{1 \times 10^{-4} \mathrm{~m}}{5 \times 10^{-11} \mathrm{~m}}=2 \times 10^{6} \text { times }=2 \text { million times }
$$

This advance in magnification has taken roughly 340 years, from 1590 to 1931.

Now, to determine the next phase of magnification if we continue to make progress in nanotechnology, we can use 1 nanometer $(\mathrm{nm})=1 \times 10^{-9} \mathrm{~m}$, and a lower limit of $.10 \mathrm{~nm}$ for such measurements. We then divide this number by (4)

$$
\frac{\text { limit of current nanoscale measurements }}{\text { limit of lengths that can be confirmed }}=\frac{1 \times 10^{-10} \mathrm{~m}}{1 \times 10^{-16} \mathrm{~m}}=1 \text { million times }
$$

The time taken to advance 2 million times in magnification made possible by microscopes, and that taken to get to the limits of nanotechnology can be compared. Nanotechnology has been around since 1959; if we have reached the limit of measurements at the nanoscale point in 60 years, then

$$
\frac{340 \text { years }}{60 \text { years }}=5.7 \text { times }
$$

In other words, we have progressed 5.7 times faster in reaching half the improvement in magnification ( 1 million times) than we did since the invention of the microscope ( 2 million times). The obvious next question is how long will it take before we get to the limit of what can be measured and confirmed?

It is instructive to compare the amount of time that it took to reach a limit of observation since the invention of the microscope, to the current limits of nanotechnology measurements, and perhaps when we encounter limits to measurement confirmation. A graph of the increases in magnification since the invention of the optical microscope shown in Figure 5 illustrates its exponential growth.

\section{(Insert Figure 5 here)}

Figure 5 The growth in magnification since the invention of the microscope shows a pattern that resembles that in Figure 1 a). When will it begin to look like that of $1 b)$ ?

Figure 5 resembles Figure 1a) albeit with a more pronounced increase; however, the question prompted by the graph is when will it begin to look like that of Figure 1b)? in other words, when will we begin to see limits to what can be measured?

Once we entertain the limits of nanoscale measurements, the region under consideration belongs to the realm of quantum mechanics, where the bizarre and fascinating features of the 
theory can serve as topics for discussion. This presents another opportunity to explore ways to engage student interest in phenomena that they may otherwise not be exposed to. Issues of causality, determinism, the role of the observer in microscopic phenomena, etc. can be effectively incorporated into the curriculum this way. The model also allows for considerations of the role of scale.

The use of the model-provided scale can help students typically under misconceptions about proportions involving the size of objects much larger than those encountered in their everyday experiences. Consider an activity where students are asked about their understanding of the scale of the size of the Earth. I have repeatedly found that many students harbor gross misunderstandings about the distances involved, when dealing with macroscopic objects, such as the size of our planet.

To make the task concrete, and relevant to their everyday experiences (since most of them drive), I have proposed the following scenario:

Imagine that we could construct a highway (at the equator, to keep the elevation fairly constant), and a person could drive around Earth. How many miles would it take to go around the Earth? The students are asked to submit their answers written anonymously so as to encourage honest individual responses.

The numbers of students who claim that one would drive millions of miles is consistently about $50 \%$ of a class of 25 students. Of this group, about half of them claim that it would take billions of miles! They seem puzzled when informed that the Moon is about a quarter of a million miles away. How can such a distorted view of the scale of the size of the Earth be entertained?

Performance on international tests of knowledge of mathematics and science given since 1995 (TIMSS reports) have demonstrated that US students exhibit a performance weakness in the areas of measurement and estimation, beginning at the earliest levels of schooling. The latest NGSS standards can really help to alleviate this by emphasizing the practice skills listed before. If undergraduate students display such recalcitrant and well entrenched misconceptions, it can be due to a number of reasons.

Misconceptions, defined as incorrect interpretations or misunderstandings of an idea, concept, or process, are often a large part of students' prior knowledge. Misconceptions can be categorized as follows (Committee on Undergraduate Science Education, 1997):

- Preconceived notions: Opinions formed without actual knowledge or experience.

- Non-scientific beliefs: opinions based on religious or mythical teachings.

- Conceptual misunderstandings: Incomplete or oversimplified knowledge from previous science courses.

- Vernacular misconceptions: Uncertainty about differences between the popular versus the scientific use of words like "work" or "theory".

- Factual misconceptions: Falsities learned at an earlier time and retained. 
The claims that having to drive billions and millions of miles to go around Earth appear to belong to at least two of the above categories. Activities that effectively incorporate the use of models such as the ones described in this essay, where students need to engage with the practices recommended by NGSS could go a long way towards improving their understanding of the role of scale and estimation in measurements. Of course, their appreciation of the proper way to look at scientific knowledge will also be a welcome outcome.

\section{Conclusion}

The use of the electromagnetic spectrum is a very viable and effective way to demonstrate many interesting features of the natural world. In this essay the focus has been on graphical representations in Figures 3, 4 and 5 that help to demonstrate the fundamental nature of scientific knowledge. The figures demonstrate science's incompleteness and its tentativeness by being subject to revision, something that many students fail to recognize and thereby see them as its strength and uniqueness. Additionally, the comparison between Figures 1 and 5 helps to broaden the context in which the role of science and technology can be graphically shown to exhibit similar patterns to the growth of human knowledge in general. Instances such as these where properties of the observable universe (although not in the human sense), can be represented with an extended version of the spectrum, can help students understand the nature of scientific knowledge in a meaningful and engaging way.

There are potentially many other ways to use modelling and creative representations of properties of the natural world, particularly those made available by interactive simulations such as the Scale of the Universe. Interestingly, such a comprehensive project was carried out by two Middle School students over a few years, which is a most commendable feat; it gives us hope that some curious young minds, despite evidence of their shortcomings in some measurements of performance in mathematics and science, will continue to explore and encourage others.

Declarations

Funding-Not Applicable

322 Conflicts of interest/Competing interests-No conflicts of interest

Availability of data and material-Not Applicable

324 Code availability-Not Applicable

325 Authors' contributions-Not Applicable

\section{References}

327

328 https://www.pewresearch.org/science/2019/08/02/trust-and-mistrust-in-americans-views-ofscientific-experts/ 
329 Arbesman, Samuel. (2012). The Half-Life of Facts. Penguin Books.

330 De Solla Price, D. J. (1965). "Networks of Scientific Papers". Science. 149 (3683): 510-515.

331 Bibcode:1965Sci...149..510D. doi:10.1126/science.149.3683.510. PMID 14325149.

332 Duncan, R. \& Weston-Smith, M. (1977). The Encyclopedia of Ignorance: everything you wanted 333 to know about the unknown. Pergamon Press.

334 Espinoza, F. (2011). The Nature of Science. Rowman \& Littlefield publishers.

335 Fuller, R. B. (1981). Critical Path. St Martins Press

336 Leydesdorff, L., Milojevic, S. (2015). Scientometrics. In Micheal Lynch (Editor), International 337 Encyclopedia of Social and Behavioral Sciences, Section 8.5: Science and Technology Studies, 338 Subsection 85030. Elsevier.

339 https://www.nextgenscience.org/sites/default/files/Why\%20K12\%20Standards\%20Matter\%20-

$340 \quad \% 20$ FINAL.pdf

341 https://en.wikipedia.org/wiki/Trends_in_International_Mathematics_and_Science_Study

342 https://www.nap.edu/initiative/committee-on-undergraduate-science-education 